\title{
RIGIDITY FOR POSITIVELY CURVED ALEXANDROV SPACES WITH BOUNDARY
}

\author{
JIAN GE AND RONGGANG LI
}

\begin{abstract}
Inspired by a recent work of Grove-Petersen in [GP18], where the authors studied Alexandrov spaces with largest possible boundary. We study Alexandrov spaces with lower curvature bound 1 and with small boundary. When the radius of $X$ is $\pi / 2$, and the boundary has diameter $\pi / 2$, we classify the total space $X$.
\end{abstract}

\section{INTRODUCTION}

Recall the radius of a metric space $(X, \mathrm{~d})$ at $p$ is defined by

$$
\operatorname{Rad}_{p}(X)=\sup \{\mathrm{d}(p, x) \mid x \in X\},
$$

and the radius of $X$ is defined by

$$
\operatorname{Rad}(X)=\inf _{p \in X} \operatorname{Rad}_{p}(X) .
$$

The celebrated Radius Sphere Theorem of Grove-Petersen [GP93] says that if $X$ is an $n$-dimensional Alexandrov space with lower curvature bound 1 and $\operatorname{Rad}(X)>$ $\pi / 2$, then $X$ is homeomorphic to a sphere. The $\pi / 2$ is sharp in the sense that the projective spaces have radius $\pi / 2$. On the other hand all positively curved Alexandrov spaces with lower curvature bound 1 can be rescaled to ones with arbitrary small radius, it is interesting to study those spaces with radius equals to $\pi / 2$. In this note we will use $\operatorname{Alex}^{n}(\kappa)$ to denote the set of all complete $n$ dimensional Alexandrov spaces with lower curvature bound $\kappa$ and use $\mathcal{M}^{n}(\kappa)$ to denote the set of all complete smooth $n$-dimensional Riemannian manifolds with lower sectional curvature bound $\kappa$. We are interested in the following set:

$$
\mathcal{A}:=\left\{X \in \operatorname{Alex}^{n}(1) \mid \operatorname{Rad}(X)=\pi / 2\right\} .
$$

For the Riemannian case, the diameter rigidity theorem of Gromoll and Grove (cf. [GG87] and [Wil01]) says that if $M$ is a smooth Riemannian manifold with sectional curvature lower bound 1 and diameter $\pi / 2$, then $M$ is either homeomorphic to the sphere or isometric to one of the Compact Rank One Symmetric Spaces (CROSS for short). Moreover, if $\operatorname{Rad}(M)=\pi / 2$, then $M$ is isometric to one of the projective spaces, i.e. $\mathbb{R P}^{n}, \mathbb{C P}^{n}, \mathbb{H P}^{n}, \mathrm{CaP}^{2}$ with the canonical metric normalized such that sectional curvatures lie in $[1,4]$.

In the Alexandrov setting, one verifies easily that for any $X, Y \in \mathcal{A}$, the spherical join $X * Y \in \mathcal{A}$. Other examples can be constructed via group actions, see discussion of rigidity part in [GP93]. Therefore, $\mathcal{A}$ contains many more spaces than

2000 Mathematics Subject Classification. Primary: 53C23, 53C20.

Key words and phrases. Alexandrov space, Riemannian manifold, Radius, Rigidity, Symmetric space. 
the Riemannian setting. It is a challenging problem to classify all such spaces. cf [RW16] for a recent rigidity result.

Inspired by a recent result of Grove-Petersen [GP18], we study the following subset of $\mathcal{A}$ :

$$
\mathcal{B}:=\left\{X \in \operatorname{Alex}^{n}(1) \mid \operatorname{Rad}(X)=\pi / 2, \partial X \in \mathcal{M}^{n-1}(1)\right\} .
$$

When we write $\partial X \in \mathcal{M}$, we consider $\partial X$ as an inner metric space in the sense that the distance between two points in $\partial X$ is the infimum of all paths connecting them in $\partial X$. Since the curvature of $X$ is positive, by [Per91], the distance function $\rho(\cdot):=\mathrm{d}(\partial X, \cdot)$ is strictly concave in $X$. Therefore there exists a unique point, denoted by $s \in X$ at which $\rho$ achieves the maximum. $s \in X$ will be called the point soul of $X$. Consequently, $X$ is homeomorphic to the cone over its boundary $\partial X$. Therefore, it is only interesting to study the geometry of such spaces.

To state our theorem, we need the following notations. For $\theta \in(0, \pi]$, the Alexandrov Lens is defined as the spherical join (cf. [GP18])

$$
L_{\theta}^{n}=\mathbf{S}^{n-2} *[0, \theta],
$$

where $\mathbf{S}^{n-2}$ is the unit $(n-2)$-dimensional sphere. The space $L_{\theta}^{n}$ admits a canonical isometric $\mathbb{Z}_{2}:=\mathbb{Z} / 2 \mathbb{Z}$ action, induced by the antipodal action on the factor $\mathbf{S}^{n-2}$ and reflection with respect to $\theta / 2$ on the other factor $[0, \theta]$. The quotient space $L_{\theta}^{n} / \mathbb{Z}_{2}$ is homeomorphic to the cone over $\mathbb{R P}^{n-1}$, with boundary isometric to the round $\mathbb{R P}^{n-1}$ of constant curvature 1 . For any $Y \in \operatorname{Alex}^{n-1}(1)$, we denote the spherical cone $[0, \pi / 2] \times{ }_{\sin (t)} Y$ by $\operatorname{Cone}^{1}(Y)$.

In order to study $\mathcal{B}$, we study the following closed related class:

$$
\mathcal{B}^{\prime}:=\left\{X \in \operatorname{Alex}^{n}(1) \mid \operatorname{Rad}_{s}(X)=\pi / 2, \partial X \in \mathcal{M}^{n-1}(1)\right\} .
$$

We have the following rigidity theorem of $\mathcal{B}^{\prime}$ :

Theorem 0.1. Let $X^{n} \in \operatorname{Alex}^{n}(1)$ with $\partial X \in \mathcal{M}^{n-1}(1)$ and $\operatorname{Rad}_{s}(X)=\pi / 2$, then

(1) ([GP18]) If $\partial X=\mathbf{S}^{n-1}$, then $X=L_{\theta}^{n}$, for $\theta \in(0, \pi]$;

(2) If $\operatorname{diam}(\partial X)<\pi / 2$, then $X=\operatorname{Cone}^{1}(\partial X)$,

(3) If $\operatorname{diam}(\partial X)=\pi / 2$ and $\partial X$ is not homeomorphic to $\mathbf{S}^{n-1}$, then the following hold:

(a) If $\partial X=\mathbb{R P}^{n-1}$, then $X=L_{\theta}^{n} / \mathbb{Z}_{2}$, for $\theta \in(0, \pi]$.

(b) If $\partial X=\mathbb{C P}^{m}, \mathbb{H P}^{m}, \mathrm{CaP}^{2}$, then $X=\operatorname{Cone}^{1}(\partial X)$.

Note that the case (1) in the theorem above is proved by Grove-Petersen in [GP18]. There is one case, i.e. $\operatorname{Rad}(\partial X) \geq \pi / 2$ except CROSSes, when we do not have rigidity result. Theorem 0.1 implies the structures of $\mathcal{B}$. If $\partial X$ is a spherical space form, we can drop the radius assumption, that is

Theorem 0.2. Let $X \in \operatorname{Alex}^{n}(1)$ with $\partial X$ isometric to an $(n-1)$-dimensional spherical space form. If $\partial X=\mathbb{R P}^{n-1}$ then $X=L_{\theta}^{n} / \mathbb{Z}_{2}$, for $\theta \in(0, \pi]$. Otherwise $X=\operatorname{Cone}^{1}(\partial X)$.

Acknowledgment: It is our great pleasure to thank Fernando Galaz-García for comments and careful reading of a preliminarily version of the paper. We also thank Luis Guijarro for his interest in our work. 


\section{Proof of the Main Theorem}

We study the subset that consists of points with maximal distance to the soul $s$ first. Let

$$
E:=\{x \in \partial X \mid \mathrm{d}(x, s)=\pi / 2\} .
$$

The set $E$ is nonempty under the hypotheses of Theorem 0.1 . The set of points on the boundary that are foot points of the soul $s$ is denoted by:

$$
A:=\{x \in \partial X \mid \mathrm{d}(s, x)=\mathrm{d}(s, \partial X)\} .
$$

The pair of sets $(E, A)$ is called dual pair of $\partial X$, which plays an important role in the study of positively curved spaces. They either coincide or are $\pi / 2$-part. When they coincide, we have the following consequence of the rigidity part from Toponogov Comparison Theorem.

Lemma 1.1. If $E=A$, then $X=\operatorname{Cone}^{1}(\partial X)$.

When $E \neq A$, we want to show they are far apart. Before proving that, let's recall: $Y \subset X$ is called $\ell$-convex if for any geodesic in $X$ with length $<\ell$ and both ends lie in $Y$ remains entirely in $Y$. Throughout this note, we call $\gamma$ a geodesic in $\partial X$, if it is locally geodesic with respect to the induced inner metric from $X$. Therefore, it is a quasigeodesic of the ambient space $X$ by the Liberman's theorem proved in [PP93]. We use $|x, y|_{X}$ or simply $|x, y|$ as shorthand for the distance between $x$ and $y$ in the metric space $X$.

Proposition 1.2. For $x \in E$ and $p \in \partial X \backslash E$. Let $\gamma:[0, \ell] \rightarrow \partial X$ be a unit speed geodesic lying in $\partial X$, connecting $p=\gamma(0)$ and $x=\gamma(\ell)$. If there exists $\uparrow_{p}^{s} \in \uparrow_{p}^{s}$ such that

then, we have

$$
\left|\uparrow_{p}^{s}, \dot{\gamma}^{+}(0)\right|=\frac{\pi}{2}
$$

$$
|x p|_{\partial X} \geq \frac{\pi}{2} \text { and }|p E|_{\partial X} \geq \frac{\pi}{2} .
$$

Proof. Applying the hinge version of Toponogov comparison theorem for the hinge based at $p$, whose sides are $\gamma$ and a geodesic from $p$ to $s$ corresponding to $\uparrow_{p}^{s}$, we get:

$$
\cos |s x| \geq \cos |s p| \cos |x p|_{\partial X}+\sin |s p| \sin \left(|x p|_{\partial X}\right) \cos \left|\uparrow_{p}^{s}, \dot{\gamma}^{+}(0)\right| .
$$

Using the condition (1.1), we have

$$
0 \geq \cos |s p| \cos (\ell)
$$

Since $p \notin E,|p s|<\pi / 2$. Therefore

$$
|x p|_{\partial X}=\ell \geq \frac{\pi}{2}
$$

It follows that $|p E|_{\partial X} \geq \frac{\pi}{2}$.

Condition (1.1) is fulfilled in many cases. For example if $E \neq A$ and thus we can find a footpoint $p \in A$ which is not in $E$. The first variational formula applied to the distance function $\rho=\mathrm{d}_{\partial X}(\cdot)$ yields $\left|\uparrow_{p}^{s}, \partial \Sigma_{p} X\right|=\frac{\pi}{2}$. Therefore (1.1) holds. We just proved:

Corollary 1.3. If $E \neq A$. Let $p \in \partial X$ be a footpoint of the soul point $s$. We have

$$
|p E|_{\partial X} \geq \frac{\pi}{2}
$$


Corollary 1.4 (Case 2 of Theorem 0.1). Let $X \in$ Alex $^{n}(1)$ with non-empty boundary $\partial X$. If diam $\partial X<\frac{\pi}{2}$ then $X$ is isometric to the spherical cone over $\partial X$

$$
X=\operatorname{Cone}^{1}(\partial X) .
$$

Proof. If $E \neq A$, then by Corollary 1.3, there exists $x \in E$ and $p \in A$ such that $|p x|_{\partial X} \geq \pi / 2$. Hence we get a contradiction to the assumption $\operatorname{diam}(\partial X)<\pi / 2$. Therefore $E=A$. By Lemma 1.1, the conclusion holds.

Next, we study the rigidity case when the lower bound of $\ell$ in Proposition 1.2 is achieved. Namely, $\ell=\pi / 2$.

Proposition 1.5. Suppose $\gamma:[0, \pi / 2] \rightarrow \partial X$ is a geodesic in $\partial X$ with $\gamma(0)=p$, satisfying $|s p|<\frac{\pi}{2}$ and $x=\gamma(\pi / 2) \in E$ with $\left|\uparrow_{p}^{s}, \dot{\gamma}^{+}(0)\right|=\frac{\pi}{2}$. Then,

$$
\left|\uparrow_{x}^{s}, \dot{\gamma}^{-}\left(\frac{\pi}{2}\right)\right|=|s p|
$$

Proof. Using the hinge version of Toponogov comparison theorem for the hinge based at $x \in E$, whose sides are geodesic connecting $x$ and $s$ and $\gamma[0, \pi / 2]$, we have:

$$
\cos |s p| \geq \cos |x s| \cos |x p|_{\partial X}+\sin |x s| \sin |x p|_{\partial X} \cos \left|\uparrow_{x}^{s}, \dot{\gamma}^{-}\left(\frac{\pi}{2}\right)\right|
$$

Using the fact $|x s|=|x p|_{\partial X}=\pi / 2$, we have

$$
\cos |s p| \geq \cos \left|\uparrow_{x}^{s}, \dot{\gamma}^{-}\left(\frac{\pi}{2}\right)\right|,
$$

which implies

$$
|s p| \leq\left|\uparrow_{x}^{s}, \dot{\gamma}^{-}\left(\frac{\pi}{2}\right)\right| .
$$

On the other hand, for any small $\epsilon>0$, using the hinge version of Toponogov comparison theorem for the hinge based at $p$, whose sides are geodesics connecting $p$ and $s$ and $\gamma[0, \pi / 2-t]$ for $t \in[0, \epsilon]$, we have

$$
\cos \left|s \gamma\left(\frac{\pi}{2}-t\right)\right| \geq \cos |s p| \cos \left(\frac{\pi}{2}-t\right)+\sin |s p| \sin \left(\frac{\pi}{2}-t\right) \cos \left|\uparrow_{p}^{s}, \dot{\gamma}^{+}(0)\right|
$$

Using the fact $\left|\uparrow_{p}^{s}, \dot{\gamma}^{+}(0)\right|=\pi / 2$, we get

$$
\cos \left|s \gamma\left(\frac{\pi}{2}-t\right)\right| \geq \cos |s p| \cos \left(\frac{\pi}{2}-t\right)
$$

that is

$$
\frac{\cos \left|s \gamma\left(\frac{\pi}{2}-t\right)\right|}{\cos \left(\frac{\pi}{2}-t\right)} \geq \cos |s p| .
$$

Therefore by the first variational formulae as $t \rightarrow 0$, we have

$$
\cos |s p| \leq \cos \left|\uparrow_{x}^{s}, \dot{\gamma}^{-}\left(\frac{\pi}{2}\right)\right| .
$$

that is:

$$
|s p| \geq\left|\uparrow_{x}^{s}, \dot{\gamma}^{-}\left(\frac{\pi}{2}\right)\right| .
$$

Combinning (1.2) and (1.3), we get

$$
|s p|=\left|\uparrow_{x}^{s}, \dot{\gamma}^{-}\left(\frac{\pi}{2}\right)\right| .
$$


Lemma 1.6 (Lemma 3.1 in [GP18]). $E$ is $\pi$-convex in $\partial X$. For any geodesic $\gamma:[0, l] \rightarrow \partial X$ that entirely lies in $E$, we have:

$$
\left|\uparrow_{\gamma(t)}^{s}, \dot{\gamma}^{ \pm}(t)\right|=\frac{\pi}{2} \quad \forall t \in[0, l]
$$

Hence by Lemma 1.6, $E \subset \partial X$ is a convex set and in particular an Alexandrov space. We want to study the dimension of $E$. The dimension puts a strong restriction on the boundary topology as we will see.

Theorem 1.7. Let $X \in \operatorname{Alex}^{n}(1)$ with boundary $\partial X$. If $\partial X$ is isometric to a compact rank one symmetric space (CROSS). Then $E$ is a closed submanifold of $\partial X$ without boundary with dimension $\geq(n-2)$.

Proof. If $\partial X=\mathbf{S}^{n-1}$, then the claim follows from [GP18]. Suppose $\partial X$ is not a sphere, then we know all the geodesics are closed in $\partial X$ with a common period $\pi$. For any $x \in E$, let $\gamma:[0, \pi] \rightarrow \partial X$ be a closed geodesic with $\gamma(0)=\gamma(\pi)=x$ in $\partial X$. Therefore, $\gamma$ is a quasigeodesic in $X$. Suppose that $\gamma$ does not lie entirely in E. Then

Claim1: $t_{0}=\pi / 2$ is the unique minimum point for the function $\mathrm{d}(s, \gamma(t))$ for $t \in[0, \pi]$, i.e.

$$
\left|\gamma\left(t_{0}\right), s\right|<|\gamma(t), s| \quad \forall t \in[0, \pi / 2) \cup(\pi / 2, \pi],
$$

Moreover

$$
\left|\uparrow_{\gamma\left(\frac{\pi}{2}\right)}^{s}, \dot{\gamma}^{+}\left(\frac{\pi}{2}\right)\right|=\left|\uparrow_{\gamma\left(\frac{\pi}{2}\right)}^{s}, \dot{\gamma}^{-}\left(\frac{\pi}{2}\right)\right|=\frac{\pi}{2} .
$$

Proof of Claim 1. Since $\gamma$ does not lie entirely in $E$, there exists $0<t_{0}<\pi$, such that $\gamma\left(t_{0}\right)$ is closest to $s$ along $\gamma$. By the first variational formula, we have

$$
\left|\dot{\gamma}^{ \pm}\left(t_{0}\right), \uparrow_{\gamma\left(t_{0}\right)}^{s}\right|=\pi / 2 \text {. }
$$

Therefore one can apply Proposition 1.2 to the triangle formed by the quasigeodesic $\gamma\left[0, t_{0}\right]$ and geodesics $p s$ and $s x$. Therefore we have $t_{0} \geq \pi / 2$. Apply Proposition 1.2 to the triangle formed by the quasigeodesic $\gamma\left[t_{0}, \pi\right]$ and geodesics $p s$ and $s x$, one get $\pi-t_{0} \geq \pi / 2$. Therefore $t_{0}=\pi / 2$.

Therefore by Proposition 1.5, we have:

$$
\left|\uparrow_{x}^{s}, \dot{\gamma}^{+}(0)\right|=\left|s \gamma\left(\frac{\pi}{2}\right)\right| .
$$

Let $\gamma:[0, \pi] \rightarrow \partial X$ be a closed geodesic with $\gamma(0)=\gamma(\pi)=x$ in $\partial X$, if $\left|\dot{\gamma}(0), \uparrow_{\gamma_{(0)}}^{s}\right|<\frac{\pi}{2}$, then by Proposition 1.5, the only point on $\gamma$ with distance $\frac{\pi}{2}$ to $s$ is $x=\gamma(0)$.

Claim 2: Let $\gamma:[0, \pi] \rightarrow \partial X$ be a closed geodesic with $\gamma(0)=\gamma(\pi)=x$ in $\partial X$. If $\left|\dot{\gamma}^{+}(0), \uparrow_{\gamma_{(0)}}^{s}\right|=\frac{\pi}{2}$, then $\gamma$ entirely lies in $E$.

Proof of Claim 3. If not, we come back to the case in Claim 1 and Claim 2, it follows that

a contradiction.

$$
\left|\uparrow_{x}^{s}, \dot{\gamma}^{+}(0)\right|=\left|s \gamma\left(\frac{\pi}{2}\right)\right|<\frac{\pi}{2},
$$

By [GP18], we know $\Sigma_{x}(X)=L^{n-1}(\theta)=\mathbf{S}^{n-3} *[0, \theta]$ for some $\theta \in(0, \pi]$. Any closed geodesic based at $x \in E$ of length $\pi$ satisfies

$$
\dot{\gamma}^{+}(0), \dot{\gamma}^{-}(\pi) \in \partial \Sigma_{x}(X) .
$$

Claim 3: $\uparrow_{x}^{s}$ is unique, i.e. $\Uparrow_{x}^{s}=\left\{\uparrow_{x}^{s}\right\}$. 
Proof of Claim 3. Fix any $\uparrow_{x}^{s} \in \uparrow_{x}^{s}$. Then any geodesic $\gamma([0, \pi])$ with $\dot{\gamma}^{+}(0)=\uparrow_{x}^{s}$ is a closed geodesic with period $\pi$ and lies entirely in $\partial X$. By (1.6), we know

$$
\left|\uparrow_{x}^{s}, \dot{\gamma}^{+}(0)\right|=\left|s \gamma\left(\frac{\pi}{2}\right)\right|=\left|\uparrow_{x}^{s}, \dot{\gamma}^{-}(0)\right| .
$$

By choosing sufficiently many initial direction of closed geodesics based at $x$, one gets $\uparrow_{x}^{s}$ has to have equal distance to any antipodal points in $\partial \Sigma_{x}(X)=\partial L_{\theta}^{n-1}=$ $\mathbf{S}^{2}$. Therefore, $\uparrow_{x}^{s}$ has to be the unique soul of $\Sigma_{x}(X)$.

By Claim 2, Claim 3 above, one get $\gamma[0, \pi] \subset E$ if and only if $\dot{\gamma}^{+}(0) \in \mathbf{S}^{n-3}$ whenever $\theta<\pi$ or $\gamma$ always lie in $E$ if $\theta=\pi$. In either case, we have $\operatorname{dim}(E) \geq n-2$. Since $E \subset \partial X$ is closed and convex, hence in its interior $E$ is locally a submanifold. Therefore, it remains to show $E$ has no boundary. This follows easily from Claim 1. In fact let $x, y \in E$ and $x \neq y$, if the closed geodesic determined by $x, y$ does not lie entirely in $E$, denoted by $\gamma[0, \pi]$, with $\gamma(0)=x, \gamma(\delta)=y$. Then by Claim 1 , there exists a unique $\gamma(\pi / 2)$ is the unique point closest to the soul $s$. Play the same game with base point $y$, one get a contradiction. Therefore if $E$ has boundary, which is necessarily convex, take $y$ to be the boundary point and $x$ be a point in $E$ that near $y$, such that the closed geodesic determined by $x$ and $y$ is normal to the boundary of $E$. The closed geodesic must leaves $E$, which contradict to we just proved.

Now we are ready to prove Theorem 0.1 Case (3).

Proof. If $\partial X=\mathbb{C P}^{m}, \mathrm{HP}^{m}, \mathrm{CaP}^{2}$, then clearly it admits no totally geodesic submanifold with codimension 1 , except the case when $\partial X=\mathbb{C P}^{1}$. However in this case if $E$ is the equator of $\mathbb{C P}^{1}$, then it leaves no room for the set $A$, since the diameter of $\mathbb{C P}^{1}$ is $\pi / 2$. Therefore by the dimension estimate in Theorem 1.7 , we know $E=\partial X$ for all the spaces listed above. Then we can apply Lemma 1.1 to get the desired rigidity $X=\operatorname{Cone}^{1}(\partial X)$.

Now we are left with the case $\partial X=\mathbb{R} \mathbb{P}^{n-1}$. In this case we have either $E=\partial X$, then by the same reason, $X=\operatorname{Cone}^{1}(\partial X)$. Or we have $E=\mathbb{R P}^{n-2} \subset \partial X$ and therefore $A=\{p\}$ the dual set of $E$ inside $\mathbb{R P}^{n-1}$. By [DGGGM18], we can take the double cover of $X$, branched over the soul point $s$, therefore we get the great sphere $\mathbf{S}^{n-2}$ as the corresponding set of maximum distance to the soul, and the set of foot points of the soul point are two points in the antipodal position on the boundary $\mathbf{S}^{n-1}$. Denote by $\theta$ the distance $|s, p|$. Then by Proposition 1.5 and Grove-Petersen's result Theorem 0.1(1), we recover the Alexandrov lens structure $L_{2 \theta}^{n} / \mathbb{Z}_{2}$. This finishes the proof.

First, we note that $\partial X$ is a space form of constant curvature 1, therefore it the unique manifold which realized the maximal volume among all Alexandrov metrics with lower curvature bound 1, then the proof goes verbatim as Proposition 1.1 in [GP18]. The authors suspect that the same conclusion holds for CROSSes, but we have no proof so far.

Proposition 1.8. Let $X \in \operatorname{Alex}^{n}(1)$ with boundary $\partial X$ isometric to the a spherical space form other than $\mathbb{R P}^{n-1}$, then

$$
\operatorname{Rad}_{s}(X)=\frac{\pi}{2} .
$$


This proposition allows us to remove the radius assumption in the main theorem. Since the space forms other than $\mathbb{R P}^{n-1}$ has no closed proper hypersurface of codimension 1, we know the set of maximum distance to the soul coincide with the set of foot points, this finishes the proof of Theorem 0.2.

\section{REFERENCES}

[DGGGM18] Qintao Deng, Fernando Galaz-García, Luis Guijarro, and Michael Munn. Threedimensional Alexandrov spaces with positive or nonnegative Ricci curvature. Potential Anal., 48(2):223-238, 2018.

[GG87] Detlef Gromoll and Karsten Grove. A generalization of Berger's rigidity theorem for positively curved manifolds. Ann. Sci. École Norm. Sup. (4), 20(2):227-239, 1987.

[GP93] Karsten Grove and Peter Petersen. A radius sphere theorem. Invent. Math., 112(3):577-583, 1993.

[GP18] Karsten Grove and Peter Petersen. A lens rigidity theorem in alexandrov geometry, arxiv:1805.10221 [math.dg]. Preprint, 2018.

[Per91] Grisha Perelman. A. D. Alexandrov's spaces with curvatures bounded from below. II. Preprint, 1991.

[PP93] G. Ya. Perelman and A. M. Petrunin. Extremal subsets in Aleksandrov spaces and the generalized Liberman theorem. Algebra i Analiz, 5(1):242-256, 1993.

[RW16] Xiaochun Rong and Yusheng Wang. Finite quotient of join in alexandrov geometry, arxiv:1609.07747 [math.mg]. Preprint, 2016.

[Wil01] Burkhard Wilking. Index parity of closed geodesics and rigidity of Hopf fibrations. Invent. Math., 144(2):281-295, 2001.

(Ge) Beijing International center for Mathematical Research, Peking University. BeIJing 100871, China

E-mail address: jge@math.pku.edu.cn

(Li) School of Mathematical Science, Peking University. Beijing 100871, China

E-mail address: lrg@pku.edu.cn 